\title{
The apparent size of the path traversed by a rotating target during saccadic and smooth pursuit: New data on the shrinking circle illusion
}

\author{
DRAKE R. BRADLEY \\ Bates College, Lewiston, Maine 04240
}

\begin{abstract}
Twelve subjects attempted to visually track a continuously or discretely moving target as it rotated through a path $20^{\circ}$ in diameter at velocities ranging from .18 to $5.2 \mathrm{cps}$. Tracking error, resulting from the eye tracking a path inside that of the target, was an increasing function of velocity from .18 to $1.13 \mathrm{cps}$. Higher velocity targets could not be tracked consistently. Apparent path diameter and the tracking diameter of the eye were both a decreasing function of velocity (through $1.13 \mathrm{cps}$ ) in the condition requiring smooth pursuit of a continuously moving target. Tracking diameter was also a decreasing function of velocity for the condition requiring saccadic pursuit of a discretely moving target, but this was not associated with a corresponding decrease in apparent size. These results suggest that the retinal position error generated by undertracking the target is processed during saccadic, but not during smooth, pursuit.
\end{abstract}

Recently, several illusory distortions have been described which arise during the visual pursuit of moving targets. For example, the apparent distance traversed by a linearly moving target is consistently underestimated during smooth pursuit (Mack \& Herman, 1972). Apparent shape distortions occur such that targets moving in an objectively square or triangular path appear to trace out paths with the sides bent inward (Festinger \& Eastman, 1974; Fujii, 1943), whereas those moving in a circular path appear to spiral inward (Hayashi, Note 1). Systematic errors in apparent spatial localization of targets smoothly pursued to a terminal position have been reported (Festinger \& Canon, 1965), as well as an apparent displacement backwards when the target comes instantaneously to a stop (Mack, Fendrich, \& Sirigatti, 1973).

The distortions described above do not arise when the stimulus display is fixated or inspected with saccadic eye movements, thus implying that such illusory effects result from special characteristics of the smooth pursuit system. In a recent paper addressing this issue, Coren, Bradley, Hoenig and Girgus (1975) report an apparent size illusion observed during the smooth pursuit of a target rotating through a circular path. As the velocity of the target is gradually increased from a slow velocity $(.18 \mathrm{cps})$

This research was conducted in partial fulfillment of the PhD requirements of the New School for Social Research. Reprint requests should be sent to Drake $\mathbf{R}$. Bradley, Department of Psychology, Bates College, Lewiston, Maine 04240. to an intermediate velocity $(1.13 \mathrm{cps})$, the path of the target appears to gradually shrink in size. Further increases in velocity result in the path expanding in size until it asymptotes at around $2.47 \mathrm{cps}$. Apparent size is nearly veridical at the very low and very high target velocities, but at intermediate velocities the size of the path is greatly underestimated.

In parametric experiments conducted on this “shrinking circle illusion," Coren et al. (1975) found that the decrease in apparent size which occurs with increasing velocity was closely related to a corresponding decrease in the diameter of the path tracked by the pursuing eye. This relationship held for those velocities where reasonably accurate visual pursuit of the target was in evidence. For higher target velocities, the eye-movement records indicated an increasing amount of saccadic involvement during attempted smooth pursuit, and this was associated with increasing apparent size. This suggests that a two-factor theory can account for the U-shaped apparent size function: (1) the apparent shrinkage in path size is caused by the pursuing eye tracking through progressively smaller paths as target velocity increases; (2) the apparent expansion in path size is caused by the increasing saccadic involvement at higher velocities, which serves to attenuate the smooth pursuit illusion.

In asserting that the path of the eye determines the apparent size of the target path, Coren et al. (1975) must assume that the position error on the retina generated by "undertracking" the target is not 
processed during smooth pursuit: otherwise the illusion cannot be derived from the contracted path of the pursuing eye. This assumption is not without some justification, as it has been shown that the smooth pursuit system is primarily a velocity matching system designed to null retinal slip velocity rather than position error (Fender, 1962; Rashbass, 1961). ${ }^{1}$ Consistent with this view is the fact that once target velocity is matched by the eye, the smooth pursuit system will often continue visual pursuit without correcting position errors which may remain (Fender, 1971; Puckett \& Steinman, 1969). When tracking sinusoidally moving targets, this situation results in a "phase lag" of the eye relative to the target, and the magnitude of this phase lag is proportional to target velocity (Dallos \& Jones, 1963; Fender, 1971; Fender \& Nye, 1961; Puckett \& Steinman, 1969; Stark, Vossius, \& Young, 1962). Under these conditions, position errors seem to be tolerated by the smooth pursuit system without any attempt at correction. In fact, Coren et al. (1975) note that subjects are not even aware they are tracking the target off-fovea. These observations support the view that the primary function of the pursuit system is to null retinal slip velocity rather than position error.

In contrast to the smooth pursuit system, the saccadic system must be capable of processing position error information in order to null image-fovea discrepancies which result from target displacement, or from the voluntary intention to fixate a different position in the visual field (Fuchs, 1971; Rashbass, 1961). For this reason, Coren et al. (1975) argued that the increasing saccadic involvement at the higher target velocities would attenuate the shrinking circle illusion, causing apparent size to eventually revert back to veridical. However, since the degree of saccadic involvement is confounded with target velocity (both increase together) as well as with other factors (fixations also increase with velocity, providing good information about the arc of the path), we cannot be certain that it is the saccades per se which are responsible for the return to veridical perception.

The role of saccades can be more readily established by devising a visual tracking task which requires saccadic pursuit of a rotating target over the same range of velocities for which Coren et al. (1975) demonstrate an apparent contraction in path size during smooth pursuit. In such a situation, we might well expect the tracking diameter of the saccadic excursions to decrease with increasing velocity, just as occurs during smooth pursuit. However, unlike smooth pursuit, the position error so generated would be processed, and therefore apparent size would not decrease with increasing velocity. The following research tests this prediction, as well as replicating the smooth pursuit condition of Coren et al. (1975). Finally, data are presented which provide an explanation as to why the eye undertracks the target during visual pursuit.

\section{METHOD}

\section{Subjects}

Twelve students, nine female and three male. served as paid volunteers in the present experiment. The median age was approximately 24 years, and all subjects had $20 / 20$ vision.

\section{Design}

A 4 by 6 factorial design with repeated measures on both factors was employed. Four viewing conditions were composed of the following: (1) continuous target movement, tracking (CT); (2) discrete target movement, tracking (DT); (3) continuous target movement, fixation (CF); (4) discrete target movement, fixation (DF). In each of these conditions, the subject viewed the target rotating at six different velocities: .18, .67, 1.13, 1.73, 2.47, and $5.2 \mathrm{cps}$. The 5.2-cps velocity generated a perceptually fused path which served as a control condition for comparing apparent size estimates at the other velocities. The fixation conditions (CF, DF) served as controls relative to the eye-movement conditions (CT, DT) in order to assess the possible effects of configurational factors on the apparent size of the paths. The CT condition elicited smooth pursuit, and the DT condition saccadic pursuit, of the target about the path. In all conditions, the dependent measure consisted of the subject's estimate of the horizontal diameter of the path traversed by the rotating target.

\section{Apparatus}

Target velocity was controlled by a Zeto-Max electric motor (Model JK3) with variable speed transmission. In the CT and CF viewing conditions, continuous target motion through a circular path, $20^{\circ}$ in diameter, was generated by a $.4-\mathrm{cm}$ diode affixed to the edge of a $29.8-\mathrm{cm}$ rotating disk. The latter was attached directly to the drive shaft of the electric motor and was viewed from a distance of $84.5 \mathrm{~cm}$. In the DT and DF conditions, discrete target movement was achieved by successively illuminating four diodes $(.4 \mathrm{~cm})$ situated at the top, left, bottom, and right sides of a path $20^{\circ}$ in horizontal and vertical extent. The circuitry, via a brush contact arrangement driven by the electric motor, was such that the "on" time of a given diode was equal to onequarter the time needed for the target to complete one revolution. Keeping the target on at a given location eliminated the tendency for the subject to smooth-track an apparently moving target between adjacent target locations. The diodes were affixed to a $76 \times 69 \mathrm{~cm}$ plywood board which could be moved in front or to one side of the rotating disk depending on whether the subject was to observe discrete vs. continuous target displacement, respectively. In addition, the plywood board doubled as an apparent size-setting device, with the distance between the two horizontally aligned diodes being adjusted by the subject via a string and pulley arrangement. All diodes were red $\left(8.57 \mathrm{~cd} / \mathrm{m}^{2}\right)$ and the direction of target rotation was counterclockwise. For the CF and DF conditions, fixation diodes were illuminated in the center of the configuration.

Electro-oculographic (EOG) recordings of eye movements through the horizontal dimension were accomplished by use of Beckman biopotential electrodes located at the outer canthus of each eye and a grounding electrode behind the right ear. The output was monitored on a Grass Model 7 oscillograph and recorded on chart paper at $25 \mathrm{~mm} / \mathrm{sec}$. The gain was adjusted such that a $10^{\circ}$ saccade from center fixation to the left or right produced a $20-\mathrm{mm}$ pen deflection up or down, sespectively, from baseline level on the chart paper. 


\section{Procedure}

After determining that the subject had adequate visual acuity, the experimenter attached the electrodes and proceeded to darkadapt the subject for a period of $20 \mathrm{~min}$. During this period general instructions were provided concerning: (1) the nature of the stimulus displays the subject would be observing (continuous, discrete); (2) the nature of the eye movements required in the different viewing conditions (smooth pursuit, saccadic pursuit, fixation); and (3) the method by which the subject would provide size estimates of the horizontal diameter of the path. In the target pursuit conditions, it was emphasized that the subject should try at all times to accurately track the target, but if this was not possible then he should keep his eyes moving counterclockwise about the path in approximately one-quarter path jumps (top to left to bottom to right, etc.).

At the end of the 20-min adaptation period, the subject was calibrated on the EOG. With head rigidly fixed in a head- and chinrest, the subject was then given two $30-\mathrm{sec}$ practice trials in the target pursuit conditions, CT and DT, at an intermediate velocity not used in the experiment proper. Then, according to the counterbalancing sequence assigned, one of the four viewing conditions was presented for the first block of trials. Each block of trials consisted of a counterbalanced sequence of the six rotational velocities of the target. For each velocity, two trials were conducted, one with a descending and one with an ascending initial position of the setting lights on the apparent size measure. Each observation period was $15 \mathrm{sec}$ in duration, at the end of which the stimulus display was replaced with the two setting lights and the subject required to estimate the apparent size of the path.

Complete eye-movement records were taken only for the CT and DT viewing conditions. For the $C F$ and DF conditions, eyemovement records were taken only for the first trial in the block to ensure that the subject was indeed fixating the center point. At the end of the block of trials for a given viewing condition, the subject was informed as to the next viewing condition to be observed and the appropriate instructions were reiterated.

\section{RESULTS AND DISCUSSION}

\section{Apparent Size Data}

The basic unit of analysis consisted of the average of the two apparent size settings (one ascending, one descending) obtained for each of the experimental conditions. Figure 1 presents the mean apparent size (deg) of the configuration as a function of target velocity and viewing condition. Inspection of Figure 1 indicates that illusory underestimation of the path occurs only at the intermediate velocities (.67-1.73 cps) of the smooth-tracking condition (CT). Analysis of variance revealed that the main effects of velocity and viewing condition were both significant, $F(5,55)=16.9, \mathrm{p}<.001 ; \mathrm{F}(3,33)=$ $9.64, \mathrm{p}<.001$, as was the Velocity by Viewing interaction, $F(15,165)=6.12, p<.001$. The magnitude of the illusion was quite strong in the smooth pursuit condition, with apparent size being underestimated by $34 \%$ at $1.13 \mathrm{cps}$.

To facilitate interpretation of the significant interaction in Figure 1, an analysis of the simple effects of each factor was conducted. Considering first the simple effect of velocity for each viewing condition, the CT condition was the only viewing condition resulting in a significant apparent size trend, $F(5,55)$ $=29.47, p<.001$. Secondly, examination of the simple effect of viewing condition at each level of the velocity factor indicated that significant effects occur only at the intermediate velocities of $.67,1.13$

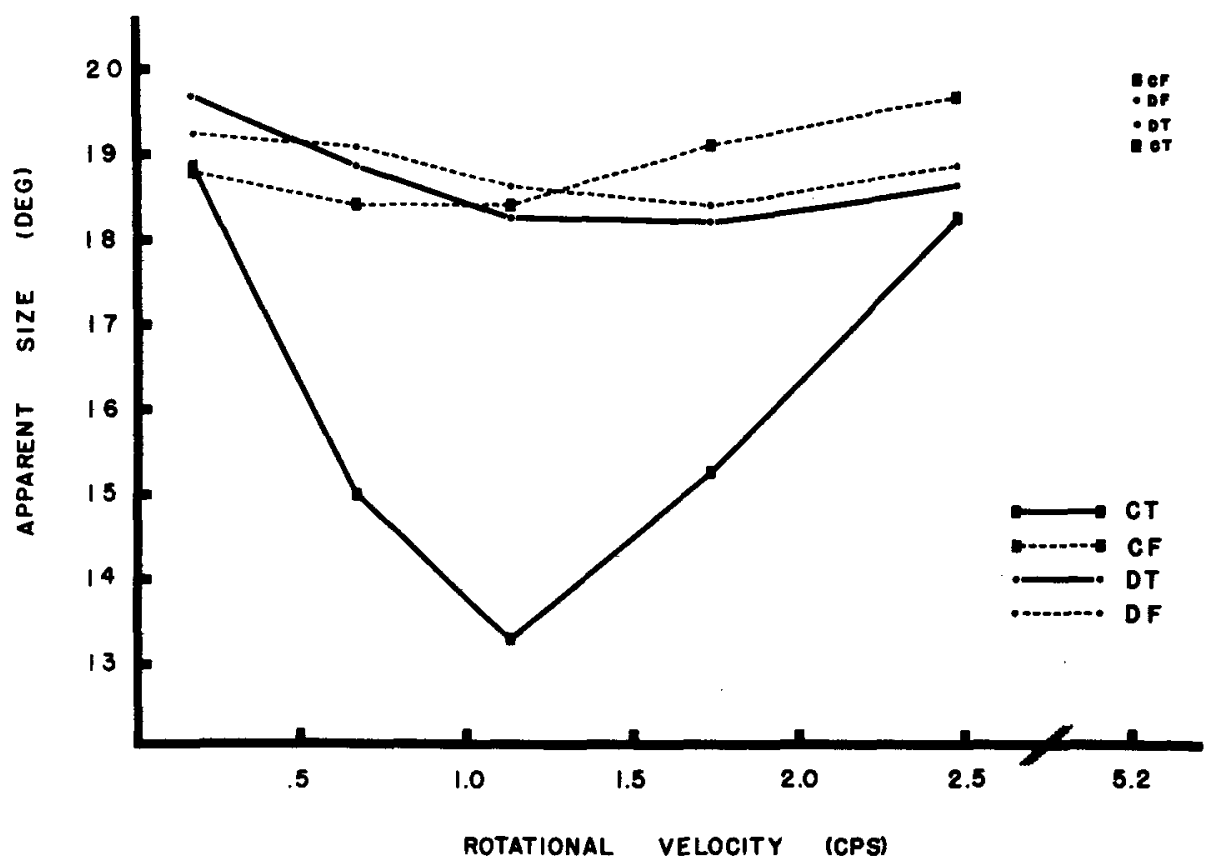

Figure 1. Apparent size-velocity functions for the smooth pursuit (CT), saccadic pursuit (DT), and fixation control (CF, DF) viewing conditions. 


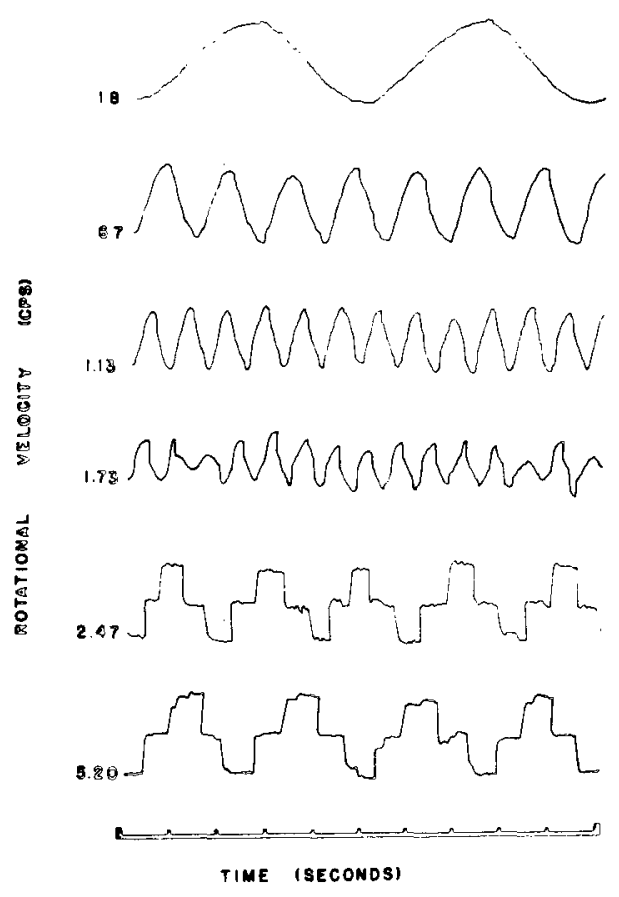

(a)

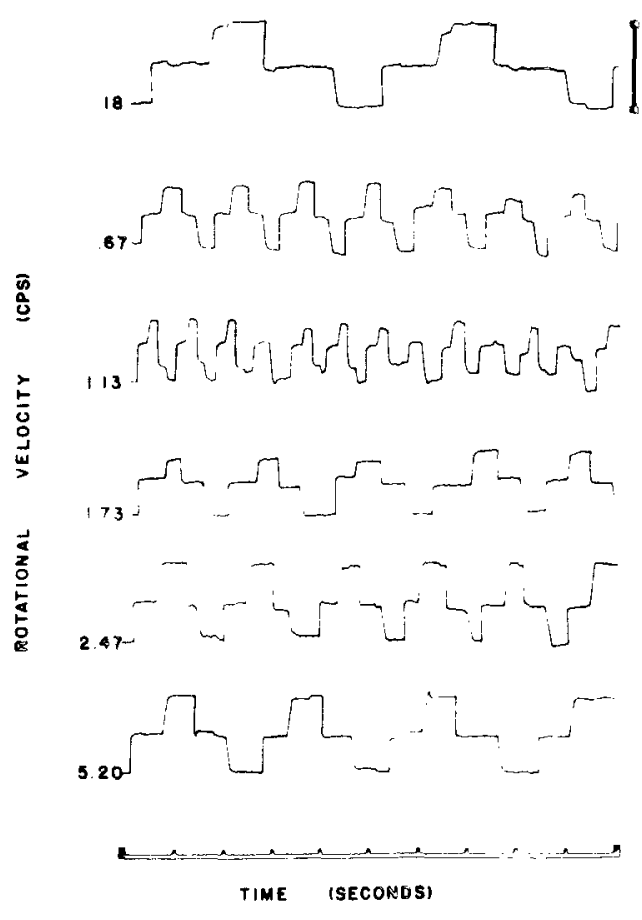

(b)

Figure 2. Eloctro-acelograph recordings of the horizontal component of eye movements during smooth pursuit (a) and saccadic purseit (b) of a rotating target.

and $1.73 \mathrm{cps}, \mathrm{F}(3,33)=4.25, \mathrm{p}<.025 ; \mathrm{F}(3,33)$ $=7.62, \mathrm{p}<.001 ; \mathrm{F}(3,33)=3.46, \mathrm{p}<.05$.

\section{Eye Movement Data}

The eye-movement data collected on the "check" trials for the CF and DF viewing conditions indicated that all subjects maintained fixation for the duration of the trial, regardless of target velocity. Figure 2 provides representative examples of EOG tracings for the CT and DT viewing conditions at each of the six rotational velocities. The pattern of EOG tracings for the smooth pursuit condition (Figure 2a) replicates that found by Coren et al. (1975), where smooth-tracking eye movements of progressively smaller diameters were evident for the .18- to 1.13-cps velocities, but thereafter begin breaking up, until the record is completely dominated by saccadic eye movements at 2.47 and $5.2 \mathrm{cps}$. The EOG tracings for the saccadic pursuit condition (Figure 2b) show that the horizontal extent of these eye movements also decreases from .18 to $1.13 \mathrm{cps}$, and thereafter increases as the subject ceases to attempt continued saccadic pursuit.

In Figures $2 a$ and $2 b$, the "regular" patterns of eye movements evident at 2.47 and $5.2 \mathrm{cps}$ result from the subject's looking about the display in onequarter path jumps, as instructed whenever it was impossible to match the rotational velocity of the target. At $1.73 \mathrm{cps}$, some subjects aitempted to maintain target pursuit in the CT and DT conditions whereas others did not. The 1.73-cps tracing of Figure $2 \mathrm{a}$ shows a subject attempting, somewhat unsuccessfully, to maintain smooth pursuit of the target. There are frequent saccadic corrections and many instances of "rephasing": i.e., very short fixations followed by a saccade operating to place the tracking eye back in phase with the target. Another subject, capable of smooth-tracking the $1.73 \mathrm{cps}$ target much more accurately, showed very few saccadic corrections and a tracking diameter which ranged between $8^{\circ}$ and $12^{\circ}$ relative to the target path diameter of $20^{\circ}$. Not surprisingly, this subject experienced maximum illusory contraction at this velocity (apparent diameter was $11.6^{\circ}$ ), rather than at $1.13 \mathrm{cps}$, the optimal illusory velocity averaging across subjects (Figure 1).

In scoring the eye-movement records, two indices were computed for each trial: the average angular sweep of the eyes through the horizontal dimension and the percent time not smooth-tracking the target (CT condition only). The first index is given by the average peak-to-trough distances (millimeter) between adjacent phases of the EOG tracings, and, when converted to deg visual angle, represents the 
average tracking diameter of the eye for that trial. the percent-time-not-tracking index is computed on the basis of the total time during a trial where smooth pursuit movements are not occurring.

Figure 3 shows, for the smooth pursuit condition, the relationship between the apparent diameter (deg) of the target path, the tracking diameter (deg) of the eyes, and the time not smooth-tracking $(\%)$, all as a function of target velocity. For the initial portion of the range, both apparent size and tracking diameter decrease as velocity increases, doing so at roughly equivalent rates. At the lowest velocity tested $(.18 \mathrm{cps})$, smooth-tracking is very accurate, with the tracking diameter of the eyes nearly matching that of the target path (i.e., $20^{\circ}$ ). However, at the optimum velocity for perceived illusion $(1.13 \mathrm{cps})$, the eye is tracking through a path with a horizontal diameter of only $13.46^{\circ}$. As velocity is increased beyond $1.13 \mathrm{cps}$, smooth pursuit movements begin breaking up as saccadic eye movements become increasingly more frequent along with intermittent visual fixations (Figure 2a). This results in the increasing time-not-tracking trend in Figure 3, which is significant by Friedman's test, $\chi_{r}^{2}(5)=58.19$, $\mathrm{p}<.001$. The decreasing tracking diameter trend is also significant, $\chi_{\mathrm{r}}^{2}(5)=47.33, \mathrm{p}<.001$.

Figure 4 shows the relationship between the apparent diameter (deg) of the target path and the tracking diameter (deg) of the eyes during saccadic pursuit, both as a function of target velocity. As in the smooth pursuit condition, tracking diameter decreases with increases in velocity up until $1.13 \mathrm{cps}$, thereafter increasing as attempted saccadic pursuit of the higher velocity targets is abandoned. The tracking diameter trend is significant by Friedman's test, $\chi_{\mathrm{r}}^{2}(5)=40.52, \mathrm{p}<.001$.

The eye-movement data show that as target velocity increases, the tracking diameter of the pursuing eye decreases in roughly comparable fashion during smooth and saccadic pursuit (Figures 2-4). This implies that the target image on the retina is positioned off center of the fovea, and that this position error increases with increases in target velocity. At $1.13 \mathrm{cps}$, the average position error of the target image relative to the center of the fovea is $3.27 \mathrm{deg}$ during smooth pursuit $[(20-13.46) / 2]$, and $3.71 \mathrm{deg}$ during saccadic pursuit $[(20-12.58) / 2]$.

\section{Implications of the Apparent Size and Eye-Movement Data}

Since a significant apparent size trend was obtained only in the CT condition (Figure 1), the shrinking circle illusion must arise from special characteristics of the smooth pursuit system. If the

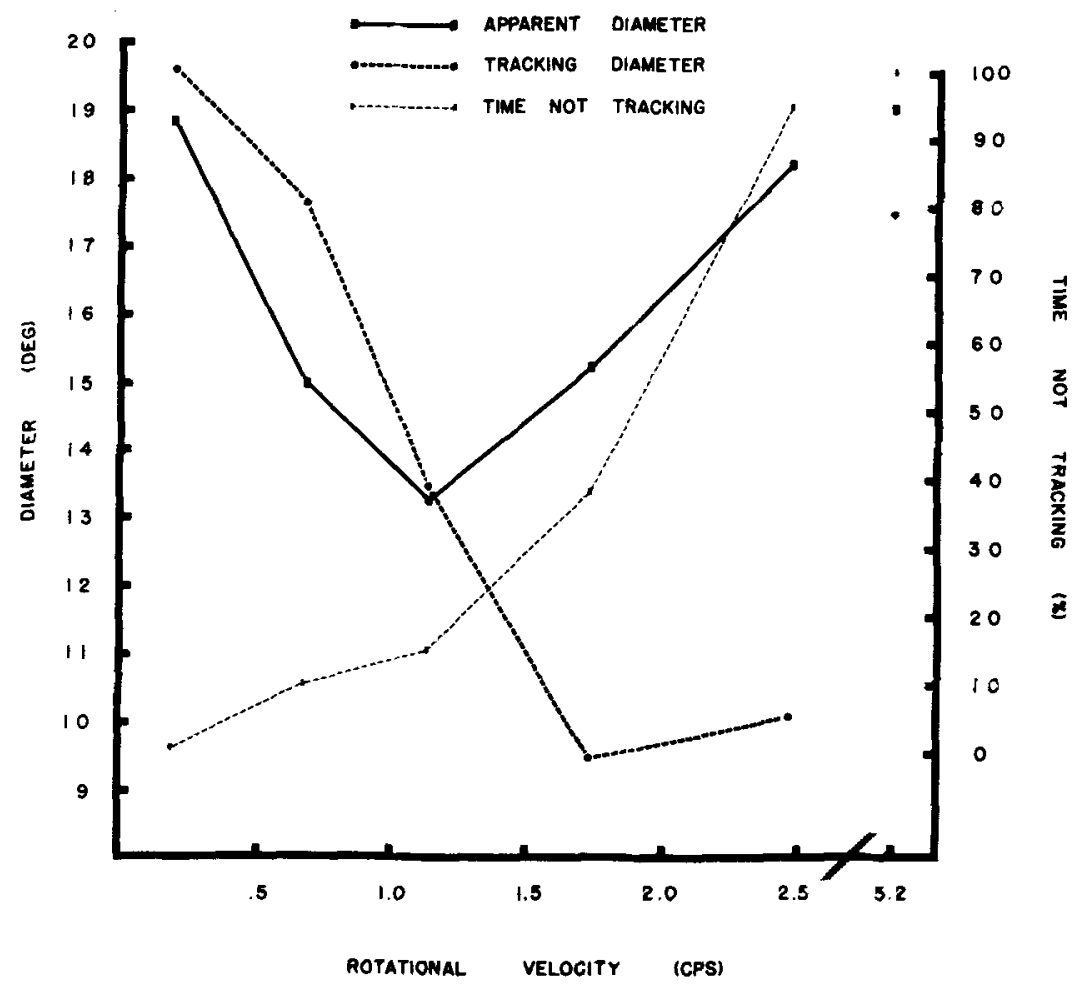

Figure 3. Apparent diameter, tracking diameter, and time not smooth-tracking as a function of target velocity during smooth pursuit. 


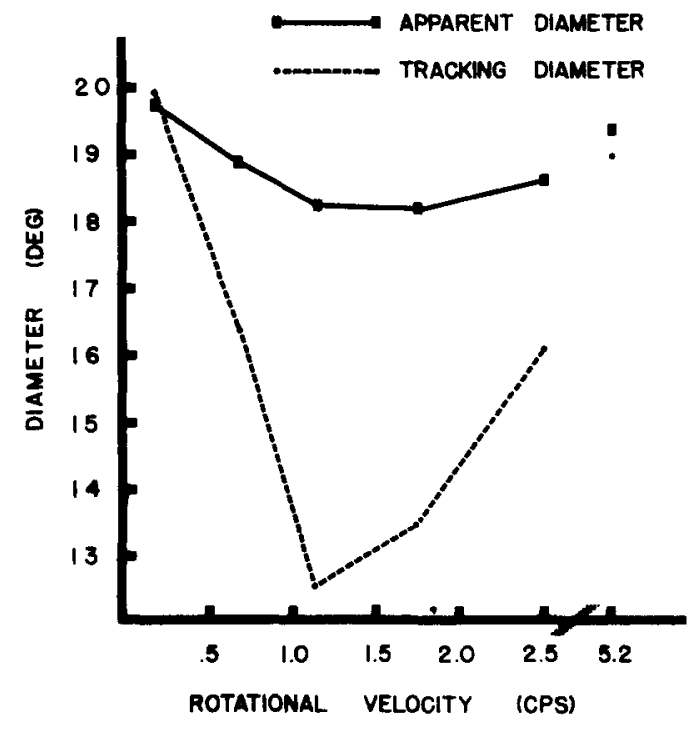

Figure 4. Apparent diameter and tracking diameter as a function of target velocity during saccadic pursuit.

illusory underestimation of path size resulted from configurational factors (Brown \& Voth, 1937) or a size-distance-velocity invariance (Day, 1969, p. 100), then the illusion should occur in other viewing conditions as well. Now, the special characteristic of the smooth pursuit system responsible for the illusion would seem to be the fact that the position errors caused by the eye tracking a path inside that of the target are not included in the estimate of path diameter. Rather, apparent diameter is quite closely related to the tracking diameter of the eye itself (Figure 3). However, position errors of equivalent magnitude during saccadic pursuit are not associated with illusory underestimation of the path diameter (Figure 4). Consequently, estimates of path diameter in this condition must depend not only on the tracking diameter of the eye itself, but also on information concerning the difference between the position of the eye and that of the target at any point on the path (i.e., position error). These results are consistent with the difference between the smooth pursuit (velocity matching) and saccadic (error nulling) eyemovement systems outlined in the introduction.

\section{Supplementary Data}

The evidence reviewed above indicates that the apparent diameter of the path during smooth pursuit is directly related to the tracking diameter of the eye. However, it is possible that some other factor causes the reduction in apparent size, and that the contracted tracking diameters simply result from efferent commands to the oculomotor system which reflect these apparent size distortions. Obviously, such an interpretation reverses the causal relations assumed here, where we have supposed that it is the contracted eye movements which, in the absence of position error information, cause the apparent size distortions. It is important, therefore, to demonstrate that the contracted tracking diameters during smooth pursuit are the result of some readily identifiable source other than the presence of preexisting apparent size distortions.

The hypothesis offered here is that the undertracking observed during smooth pursuit results from a physical limitation of the eye in matching target velocity. A number of investigators have established that the maximum attainable smooth pursuit velocity is between 30 and $40 \mathrm{deg} / \mathrm{sec}$ angular velocity (Dodge \& Cline, 1901; Stark, 1971; Westheimer, 1954; Young, 1971). Since even the $.67 \mathrm{cps}$ condition exceeds this limit (the target has an angular velocity of approximately $42 \mathrm{deg} / \mathrm{sec}$ ), we see that the eye will not be able to match target velocity if it tracks through the same angular extent as the target. ${ }^{2}$ The eye can, however, reduce its angular velocity by reducing its tracking diameter, since this decreases the circumference of the path tracked in a given rotation. Specifically, we might expect that the eye will reduce its tracking diameter just enough so that it can match the rotational velocity of the target while still maintaining smooth pursuit at an angular velocity equal to or just below its upper limit. If this view is correct, then computations of the angular velocity of the eye based on the peak-to-trough distances in the EOG records (and correcting for saccadic velocity contamination) ought to yield values in the vicinity of $30-40 \mathrm{deg} / \mathrm{sec}$ in all conditions where the target velocity exceeds this limit. ${ }^{3}$ Such computations are meaningful only for these conditions where all, or at least some, subjects are capable of consistently maintaining smooth pursuit of the target (i.e., .18$1.73 \mathrm{cps}$ ).

To test the above prediction, several representative eye-movement recordings of subjects smooth tracking . 18-1.73-cps-velocity targets through a path $20.5^{\circ}$ in diameter were analyzed from data collected by Bradley (1974). The results of this analysis are presented in Figure 5, where the angular velocity of the target and eye are each plotted as a function of the rotational velocity of the target. Note that the angular velocity of the eye matches that of the target for velocities up to $37 \mathrm{deg} / \mathrm{sec}$ (this statement is based on interpolation), whereas for target velocities exceeding this, the angular velocity of the eye asymptotes within the range defining its upper limit $(30-40 \mathrm{deg} / \mathrm{sec})$. Clearly, this is possible only if the eye tracks through progressively smaller paths at the higher target velocities. In fact, it appears that the increasing amounts of undertracking observed from .67 to $1.73 \mathrm{cps}$ (Figure 3 ) may be entirely accounted for by assuming that the eye tracks through whatever size path is necessary to maintain smooth pursuit 


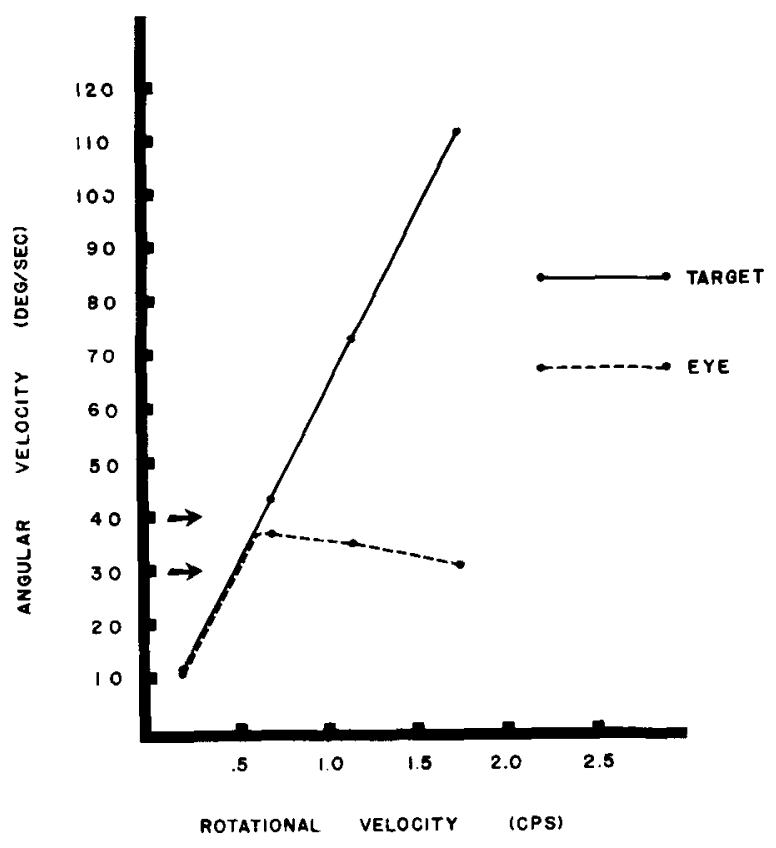

Figure 5. Angular velocity of target and eye as a function of target rotational velocity.

while still matching the rotational velocity of the target. If so, we should be able to independently predict the tracking diameter of the eye for any given rotational velocity by assuming that it is always limited to an upper pursuit velocity of around $35 \mathrm{deg} / \mathrm{sec}$. When this is done, the computed values are quite close to the observed values plotted in Figure 3 (correcting for the effect of saccades). These findings suggest that the contracted tracking diameters derive not from configurational or stimulus factors, but rather from a physically determined limit of the eye in pursuing rapidly moving targets. Consequently, the argument that the illusory distortion causes the contracted eye movements, rather than the reverse, would seem untenable. ${ }^{4}$

\section{Conclusion}

The present research shows that tracking error during smooth pursuit is associated with illusory underestimation of the apparent diameter of the target path. Tracking error during saccadic pursuit is not associated with illusory underestimation, even though the magnitude of this error is approximately the same as that observed during smooth pursuit. It is suggested that because the smooth pursuit system is a velocity matching system, retinal position errors may not be processed during visual pursuit. If so, apparent size distortions should result whenever the eye undertracks the target. Such tracking errors occur with increasing target velocity simply because the eye cannot exceed $40 \mathrm{deg} / \mathrm{sec}$ angular velocity during smooth pursuit. As target velocity exceeds this limit, the eye tracks through progressively smaller paths in order to maintain smooth pursuit at or below $40 \mathrm{deg} / \mathrm{sec}$, and this in turn results in the apparent shrinkage in path diameter.

\section{REFERENCE NOTE}

1. Hayashi, K. The apparent path of a circularly moving spot. Fifth Research Report. Psychological Laboratory on the Hiyoshi Campus, Keio University, Japan, May 1971.

\section{REFERENCES}

Bradley, D. R. The circle illusion: An analysis of the effects of eye movements on the apparent size of the path traversed by a circularly moving target. (Doctoral dissertation, New School for Social Research, 1973). Dissertation Abstracts International, 1974, 34, 3518-B.

Brown, J. F., \& Voth, A. C. The path of seen movement as a function of the vector field. American Journal of Psychology, 1937, 49, 543-563.

Coren, S., Bradley, D. R., Hoenig, P., \& Girgus, J. S. The effect of smooth tracking and saccadic eye movements on the perception of size: The shrinking circle illusion. Vision Research, 1975, 15, 49.55.

Dallos, P., \& Jones, R. Learning behavior of the eye fixation control system. IEEE Transactions, 1963, AC8, 218-227.

DAY, R. H. Human perception. New York: Wiley, 1969.

Dodge, R., \& Cline, T. S. The angle velocity of eye movements. Psychological Review, 1901, 8, 145-147.

FENDER, D. H. The eye movement control system: Evolution of a model. In R. F. Reiss (Ed.), Neural theory and modeling (Proceedings Ojai Symposium). Stanford: Stanford University Press, 1962.

Fender, D. H. Time delays in the human eye-tracking system. In P. Bach-y-Rita, C. C. Collins, \& J. E. Hyde (Eds.), The control of eye movements. New York: Academic Press, 1971. Pp. 539-543.

FENDER, D. H., \& NYE, P. W. An investigation of the mechanics of eye movement control. Kybernetik, 1961, 1, 81.

Festinger, L., \& Canon, L. K. Information about spatial location based on knowledge about efference. Psychological Review, 1965, 72, 373-384.

Festinger, L., \& Eastman, M. Inferences about the efferent system based on a perceptual illusion produced by eye movements. Psychological Review, 1974, 81, 44-58.

Fuchs, A. F. The saccadic system. In P. Bach-y-Rita, C. C. Collins. \& J. E. Hyde (Eds.), The control of eye movements. New York: Academic Press, 1971. Pp. 343-362.

FurI, E. Forming a figure by movement of a luminous point. Japanese Journal of Psychology, 1943, 18, 196-232.

MaCK. A., \& Herman, E. A new illusion: The underestimation of distance during pursuit eye movements. Perception \& Psychophysics, 1972, 12, 471-473.

Mack, A., Fendrich, R., \& Sirigatti, S. A rebound illusion in visual tracking. American Joumal of Psychology, 1973, 86, 425-433.

Puckett, J. deW., \& STeinman, R. M. Tracking eye movements with and without saccadic control. Vision Research, 1969, 9. 695-702.

Rashbass. C. The relationship between saccadic and smooth tracking eye movements. Journal of Physiology, 1961, 159. 326-338.

Rock. I., \& Halper, F. Form perception without a retinal image. American Journal of Psychology, 1969, 82, 425-440.

STARK, L. The control system for versional eye movements. In P. Bach-y-Rita, C. C. Collins, \& J. E. Hyde (Eds.), The control of eye movements. New York: Academic Press. 1971. Pp. 363-428. 
Stark, L., Vossius, G., \& Young, L. Predictive control of tracking eye movements. IRE Transactions, 1962, HFE3, 52-57.

Westheimer. G. H. Eye movement responses to a horizontally moving visual stimulus. Archives of Ophthalmology, 1954, 52, 9.32-943.

Young, L. R. Pursuit eye tracking movements. In P. Bach-y-Rita, C. C. Collins, \& J. E. Hyde (Eds.). The control of eye movements. New York: Academic Press. 1971. Pp. 429-443.

\section{NOTES}

1. There is no clear consensus as to the nature of the information available during smooth pursuit, although most investigators agree that retinal slip velocity is the primary input to the system. Festinger and his colleagues have argued that information about eye position is poor or nonexistent during smooth pursuit, although information about the velocity and direction of the eye movement is available (Festinger \& Canon, 1965). If a record of the path tracked by the eye during smooth pursuit is not maintained, however, it is difficult to understand how subjects are able to successfully identify the shape of the path (Rock \& Halper, 1969), or why indeed they have any impression whatsoever of the size and shape of the path traversed by a circularly rotating target (Coren et al., 1975). Perhaps more troubling for the present argument is that Festinger and Eastman (1974) have assumed that retinal position error information is processed during smooth pursuit. Based on this and other assumptions, they successfully predicted the apparent shape of the path traversed by a target moving in an objectively square path. However, if we use the same assumptions to predict what will be seen during the smooth pursuit of a circularly rotating target, we find that apparent size should increase with increasing velocity (and therefore position error), rather than decrease. Consequently, we will assume that the visual system maintains a record of the path tracked during smooth pursuit, and that estimates of apparent path diameter are based only on this information: i.e., a record of the position errors occurring during smooth pursuit is not available.

2. For circularly rotating targets, angular velocity (deg/sec) equals $\pi$ times the diameter of the path (deg) times the rotational velocity (cps).

3. The angular velocity of the eye is computed in the same manner as that of the target, except that the tracking diameter of the eye is used in the equation rather than the path diameter. However, using the average peak-to-trough distance in the EOG tracing (Figure 2a) will result in inflated estimates of the angular velocity of the eye, because some of the tracking is actually being achieved by high-velocity saccades rather than by continuous smooth pursuit. To correct for saccadic velocity contamination, the average peak-to-trough component in the EOG tracing due to saccades is subtracted from the average overall peak-to-trough distance, and this "adjusted" tracking diameter is then used to compute the angular velocity of the eye. Finally, note that these computations require the assumption that the tracking diameter of the eye in the vertical dimension is approximately the same as that measured in the horizontal dimension.

4. The eye-movement record used to compute the angular velocity of the eye at $1.73 \mathrm{cps}$ (Figure 5) was obviously not a typical record for that velocity, since relatively few subjects were capable of maintaining accurate smooth pursuit for the duration of the trial (although many could do so for short periods). Nevertheless, for those subjects that did, we see that the angular velocity of the eye is between 30 and $40 \mathrm{deg} / \mathrm{sec}$, as predicted. Furthermore, these subjects tended to perceive maximum illusory contraction at $1.73 \mathrm{cps}$ (see example cited in results). This would be expected, since the tracking diameter of the eye is at a minimum at this velocity and the saccadic involvement, for these particular subjects, is quite low. This finding also helps to explain an apparent anomaly in the data plotted in Figure 3: Since the time not tracking is substantial at $1.73 \mathrm{cps}(38 \%)$, we might expect that the apparent diameter of the path should show less underestimation than at $.67 \mathrm{cps}$, where the saccadic involvement is minimal. In fact, apparent diameter is about the same at .67 and 1.73 cps. However, when smooth pursuit is successfully accomplished at $1.73 \mathrm{cps}$, the tracking diameter of the eye is much smaller than it is at $.67 \mathrm{cps}$. Hence, the increased saccadic involvement, which generates a veridical impression of path diameter, may be offset by periods of successful smooth pursuit where the diameter of the path appears quite small. The subject, faced with these conflicting sources of information during the 15 -sec trial, probably performs a crude averaging operation, pooling the two sources of information in an attempt to provide a representative estimate of the path diameter for that trial. Likewise, averaging across subjects, some of whom perceive the path as veridical (poor trackers) and some of whom perceive it as very small (good trackers), would create the same intermediate result in apparent size.

(Received for publication January 8, 1977; revision accepted June 1, 1977.) 